\title{
Cervical Ectopic Pregnancy: A Puzzling Event
}

Shahana Begum ${ }^{1 *}$

Afroza Ferdous ${ }^{1}$

Munawar Sultana'

Rowshan Ara Begum'

'Department of Obstetrics \& Gynaecology Chittagong Medical College

Chittagong, Bangladesh.
${ }^{*}$ Correspondence to:

\section{Dr. Shahana Begum}

Junior Consultant

Department of Obstetrics \& Gynaecology

Chittagong Medical College

Chittagong, Bangladesh.

Mobile: +8801714288794

E-mail: drshahanashelly@yahoo.com

\begin{abstract}
Cervical ectopic pregnancy is the implantation of the conceptus within the cervix below the level of internal os ${ }^{1}$. Such pregnancy typically aborts within the first trimester, if it is implanted closer to the uterine cavity called cervico isthmic pregnancy it may continue longer ${ }^{2}$. Cervical pregnancy accounts for less than $1 \%$ of all ectopic pregnancies, with an estimated incidence of 1 in 2500 to 1 in 18000 . Though the pregnancy in this area is uncommon but possibly life threatening condition due to risk of severe hemorrhage and may need hysterectomy ${ }^{2,3}$. Early detection and conservative approach of treatment limit the morbidity and preserve fertility. A 37 year old lady para $4+0$ diagnosed as a case of cervical ectopic pregnancy with intractable bleeding and save the patient by emergency hysterectomy to control hemorrhage. The case is reported here for its relative rarity.
\end{abstract}

Key words: Cervical ectopic pregnancy; methotrexate (MTX); intractable bleeding; emergency hysterectomy.

\section{INTRODUCTION}

In ectopic pregnancy the fertilized ovum becomes implanted in a site other than normal uterine cavity. It is the consequence of an abnormal implantation of the blastocyst ${ }^{1}$. Worldwide incidence of ectopic pregnancy is $3-4 \%$ but the incidence is rising. In some studies the incidence reported as high as 19.7 ectopic pregnancies for 1000. It is one of the leading cause of maternal mortality. About $95-98 \%$ of ectopic pregnancy occur in fallopian tube. Ectopic pregnancy in cervix is rare but occasionally encountered in obstetric practice. The common characteristic point is implantation into myometrial defects following previous intrauterine surgery like previous attempts of cervical dilation, previous history of caesarean section ${ }^{2}$. As a result of myometrial involvement, surgical evacuation of cervical ectopics often results in severe haomorrhage. The bleeding tends to be more severe with increasing gestational age. treatment options depend on patient's condition, gestational age, fertility status ${ }^{3}$. Cervical ectopic can be easily misdiagnosed as a threatened abortion and rupture may lead to potentially fatal bleeding. An attempt to remove cervical pregnancy is likely to cause severe vaginal bleeding , and hysterectomy rates of $40 \%$ have been described when dilatation and curettage was attempted without preoperative diagnosis of cervical pregnancy.

So obstetrician must suspect cervical ectopic pregnancy in women with first trimester vaginal bleeding in order to permit early diagnosis and fertility saving treatment. 


\section{CASE REPORT}

A female patient, age of 37 years lady para $4+0$, age of her last child was 3 years all were delivered at home, hailing from Bashkhali, Chittagong was admitted into Chittagong Medical College Hospital (CMCH) on 27 march 2014 as an emergency patient as a case of cervical fibroid with severe anaemia. Regarding her menstrual cycle she was a regularly menstruating women with average flow and duration. She had history of irregular intake of oral contraceptives pill. She was suffering from irregular per vaginal bleeding for last 3 month and went to local doctor for several times. according to her physicians advice she had an ultrasonography of lower abdomen. The report revealed that there is a big cervical fibroid and referred to $\mathrm{CMCH}$ for better management. On admission her body build was average but she was severely anaemic and patient was haemodynamically stable. Per abdominal finding revealed uterus was 16 weeks size, firm in consistency, per vaginal examination cervix was patulous but broad and hypertrophied and bleeding was minimum. Her haemoglobin percentage was $6.6 \mathrm{gm} \%$, initially patient was transfused 4 unit of blood. Again advised for ultasonography of her lower abdomen for further evaluation. The report shows that there was a big cervical growth and advised for examination under anaesthesia and biopsy. On $2^{\text {nd }}$ april 2014 during examination under anaesthesia it seems to be a case of incomplete abortion and then attempted for evacuation, during evacuation initially some amount of product of conception was expelled out with sponge holding forceps then there was intractable bleeding was started. After exclusion of perforation, inspite of all uterotonic drugs and continuous uterine massage bleeding was not controlled then immediate decision was taken for laparotomy.

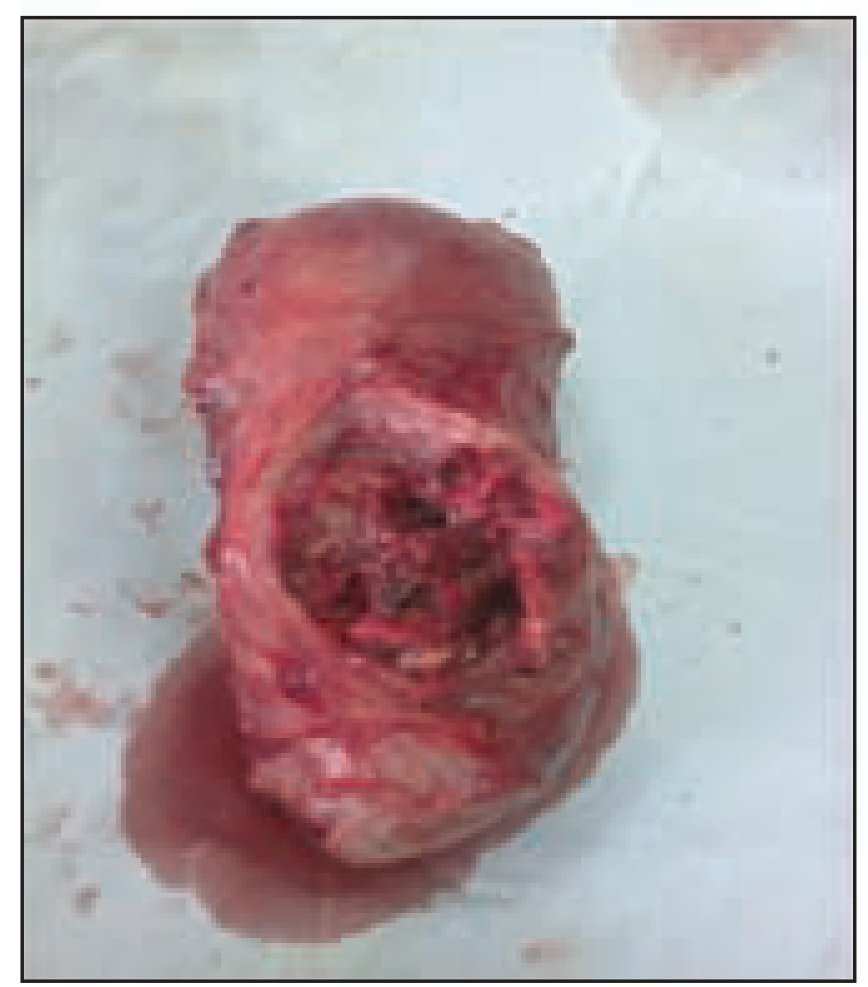

Figure 1: Product of conception within endocervical region.

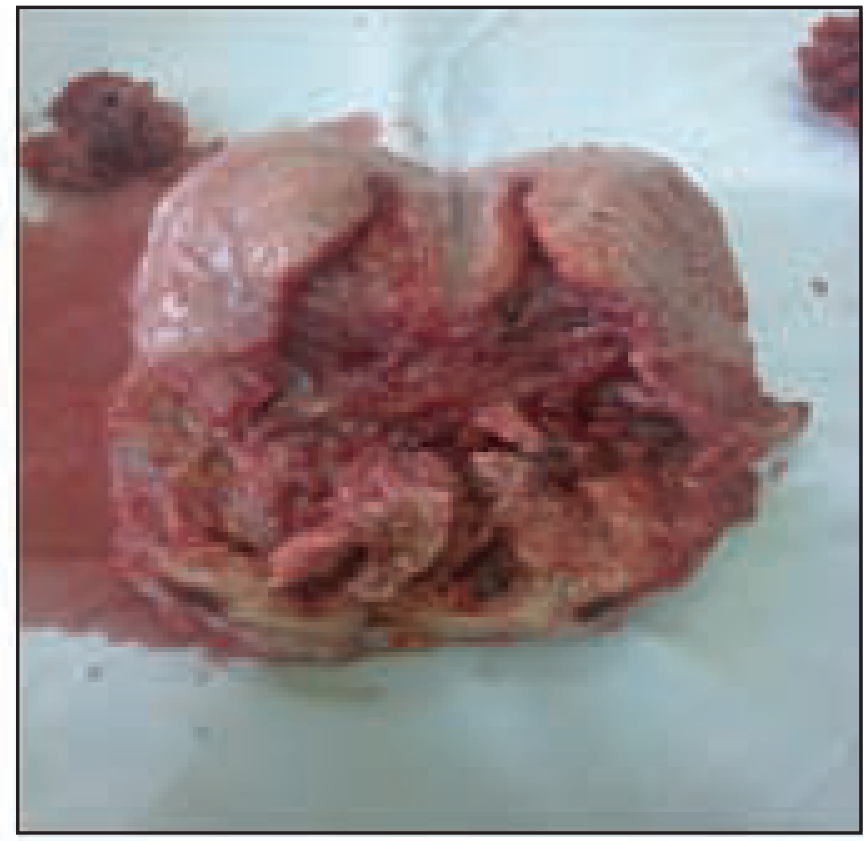

Figure 2: Endometrial cavity is empty.

On laparotomy findings body of the uterus looks little bit bulky lies above the distended cervix. Whole uterus was 16 weeks size, cervix was distended seems to be full with something within it and fundus was compatable with cervix with a narrowing in between them. Decision was taken for hysterectomy, After giving first clamp and vesico uterine peritoneum was dissected out then a vertical incision was given over the cervix, moderate amount of product of conception was seen within the endocervical region, it was removed digitally and diagnosed as a case of cervical pregnancy. Bleeding was continuing and total abdominal hysterectomy was done without any peroperative difficulties. Time of operation was 45 minutes. Two units of fresh blood were transfused during operation. Tissue was sent for histopathological examination. Report showed chorionic villi within endocervical region and secretory changes within the endometrium. No cells of granuloma or malignancy were seen. Her serum hCG level was less than 1. Postoperatively patient was recovered well and discharged from hospital on $7^{\text {th }}$ postoperative day.

\section{DISCUSSION}

Although the exact etiology of cervical ectopic pregnancy was unknown, but the probable predisposing factors are accelerated migration of fertilized ovum to the cervical canal before it is capable of nidation, damage of the endocervical canal due to previous instrumentation e.g prior dilation and curettage, PID, anatomical anomalies (myomas, synaechiae), use of intrauterine device,in vitro fertilization (IVF), diethylstilbesterol exposure is the another factor ${ }^{4-7}$.

Classically these patients presents with first trimester per vaginal bleeding, with or without abdominal pain, pelvic pain, haemorrhagic mass, a gestational sac with the presence of an embryo with or without cardiac activity ${ }^{8}$. Per speculum examination reveals a partially open external os, blood mucoid 
discharge from cervix, a visible cervical lesion, bulky bluish cervix with a fluctuant purple or blue mass; or cervix may be distended or oedematous in appearance ${ }^{9}$. In our case vaginal examination revealed soft cervix that was disproportionately enlarged compared with the uterus and profuse hemorrhage occurred during manipulation of the cervix. Uterus is usually 6 8 weeks size in cervical pregnancy,with unremarkable adnexae or tenderness may be present ${ }^{10-11}$. In our case it was 16 weeks in size due to some adenomyomatous changes within the myometrium. For accurate diagnosis sonographer must be familiar with distinction between cervical pregnancy,cervical dilatation and early intrauterine pregnancy ${ }^{12-13}$.

In the past, the diagnosis of cervical pregnancy was made primarily at the time of histological analysis of hysterectomised uterus. Cervical pregnancy was first described in 1817 and named as such in $1860^{14}$. In 1911, Rubin proposed the pathological criteria for the diagnosis of cervical ectopic pregnancy. There were cervical gland must be present opposite the placental attachment; the attachment of the placenta to the cervix must be intimate. The whole or a portion of the placenta must be situated below the entrance of the uterine vessels or below the peritoneal reflection of the anterior or posterior wall of the uterus and no fetal elements seen in the corpus uteri. First reported case of cervical case was diagnosed by ultrasonography in $1978^{15}$. In 1993 Timor-tritsch proposed some tringent criteria ${ }^{16}$. the placenta and entire chorionic sac containing the live pregnancy must be below the internal os, cervical canal must be dilated and barrel shaped. Gestational sac in cervical abortion often crenated but in cervical pregnancy it become round or oval and demonstrate some fetus or yolk $\mathrm{sac}^{17}$. Internal os invariably remain dilated in cervical abortion and completely closed in cervical pregnancy. Endometrial cavity often shows a dense echogenic mass representing a mixture of blood and product of conception, body of the uterus being larger than the non gravid state owing to recent loss of intrauterine sac.while in the cervical pregnancy, prominent endometrial echo caused by decidual reaction ususlly seen. In 1996, jurkovic proposed sliding sign detected on TVS examination while gentle pressure is applied on the cervix with the probe, the gestational sac of an abortus slides against the endocervical canal, this sliding motion usually could not be found in implanted cervical pregnancy ${ }^{18}$. Color Dopper can show peritrophoblastic blood flow from an active vascular supply to the conceptus. The nonviable sac transiently passing through the cervix,will not have any peritrophoblastic flow, as it separated from its vascular supply ${ }^{19}$.

Traditionally these pregnancies were managed surgically.Due to anatomical location, surgery is notoriously hadarzous.consequenty,many women with this type of problem underwent hysterectomy .Now there has been significant shift from such an approach to more conservative treatment modalities. Due to improved access to TVS scanning and rapid assay of serum bhcg, most ectopics, including cervical pregnancies are now detected earlier. As a result of diagnosis earliar in gestation, women have serum hCG levels and are clinically more stable, therefore can be offered conservatives management ${ }^{20}$. Use of anti metebolite MTX has facilitated the medical management. conservative treatment is expectant and medical. Expeatant management is a 'wait and see' approach. medical managements in the from of systemic or intraamniotic MTX, with or without intra-amniotic potassium chloride administration criteria for conservative treatment are patient should be haemodynamically stable, have no haemoperitonium on TVS scanning, have normal kidney and liver function ${ }^{21}$. Additional specific requirements for expectant managemants are no fetal cardiac activity ${ }^{22}$. Serum hCG level were measured on day 1, 4 and 7 with day 1 being the day of first injection of MTX. If serum Bhcg level drop by more than $15 \%$ between day 4 and 7 , this deemed acceptable. Serum hCG level should be checked weekly until $<10 \mathrm{iu} / \mathrm{L}^{23}$. Cervical pregnancies with fetal cardiac activity, initial treatment should idealy be local MTX or potassium chloride injection with or without interval curettage. Multidose, alternate day systemic MTX is an alternative treatments $(1 \mathrm{mg}$ $/ \mathrm{kg}$ on day1, 3 and 5)with folinic acid rescue $(0.1 \mathrm{mg} / \mathrm{kg}$ on day 24 and 6).For intraamniotic administration 16 guage double lumen oocyte collection needle can be introduced under TVS guidence into the amniotic sac and TVS is repeated on alternative day to check viability.if fetal cardiac activity is persisted intraamniotic potassium chloride is injected. The amniotic sac must be kept intact to avoid substantial bleeding.Cause of MTX failure are presence of fetal cardiac activity, initial serum gCG level $>10,000 \mathrm{IU} / \mathrm{L}$, gestational sac more than 9 weeks, crown rump lelgth more than $10 \mathrm{~mm}$ if the treatment fail both the single and multiple dose of MTX, intra amniotic injection of potassium chloride will be successful ${ }^{24}$.

Surgical treatment are conservative surgery and definitve surgery. Conservative surgery is curettage but it may be combined with other mechanical techniques to reduce blood supply. Reduction of blood supply by cervical circlage,ligation of cervical arteries, uterine artery, internal artery and angiographic embolisation of the cervical, uterine or internal illiac arteries. These procedure has recently been used primarily as rescue therapy when profuse bleeding follows ${ }^{25}$. Intracervical vasopressin has also been used to minimize bleeding. Curettage is used to remove sonographically nonviable pregnancy efter chemotherapy. some believe that curettage is necessary to reduce massive haemorrhage after trophoblastic shedding from the atonic cervix that occurs as a metabolic effect of MTX $^{24-25}$.

Cervical or vaginal packing was most commonly used to apply direct pressure on the bleeding site. Use of Foleys cather, with inflation of baloon within the cervical canal is more effective is achieving haemostasis than packing ${ }^{26}$. Definite surgery is hysterectomy, indication of which are intractable haomorrhage, second or third trimester cervical pregnancy and no desire for fertility.

Outcome of conservative treatment of cervical pregnancy is promosing. A review of English literature from 1911 to1994 found 39 cervical ectopic pregnancies and 29 patient were treated conservatively ${ }^{27}$. Out come of most of the pregnancies after cervical ectopic will lead to term deliveries but there is possibility of preterm labour in such $\operatorname{cases}^{22-23}$. 


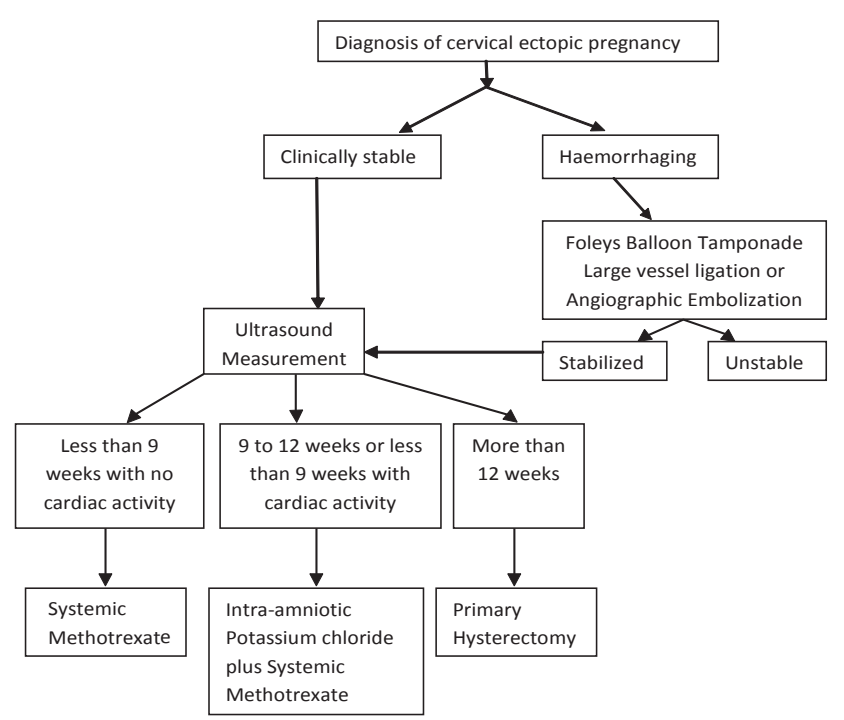

Figure 3: Suggested treatment algorithm of cervical ectopic pregnancy

\section{CONCLUSION}

A cervical ectopic can easily be misdiagnosed as threatened spontaneous abortion, the distinction is extremely important ,as the treatment methods differ significantly. Simple speculum examination is found to be more informative and less likely to result in bleeding than bimanual examination.

It is important to assess both the patient and services available while making treatment decisions. Methotrexate is reasonable first line therapy. It is easy to administer and can be given in any hospital. Gynaecologist should be highly aware of these type of cases and should be skilled enough in the diagnosis and management of this life threatening condition.

\section{DISCLOSURE}

All the authors declared no competing interest.

\section{REFERENCES}

1. Lin EP, Bhalt S, DograVS. Diagnostic clue of ectopic pregnancy.Radiographics 2008;28 (6):1661-1675.

2. Avery DM, Wells HA, Harper DM. Cervico-isthmic corporeal pregnancy with delivery at term: A review of literature with a case report.Obstet Gynaecol Surv 2009;64(5):335-344.

3. Leeman LM, Wendland CL. Cervical ectopic pregnancy, Diagnosis with endocervical ultrasound examination and successful treatment with methotrexate. Arch from Med.2000;9:72-79.

4. Michele G Sullivan, Elsevier global Medical news, Cervical ectopic pregnancy easy to miss, ACEP news, February 2010.

5. Studdiford WE, Cervical pregnancy; A review of literature and report of two probable caeses,Am J Obstet Gynaecol 1945:49: 169-185.

6. Shinaganwa S, Na gayama M, Cervical pregnancy as a possible sequele of induced abortion. Am J Obstet Gynaecol. 1969;105:282-284.

7. Rothe Dj, Birnbaum SJ,Cervical pregnancy, Obstet Gynaecol 1973; 42:675-680.

8. Cepnil I, Okal P, Erkan S,Erzik B. Conservative treatment of cervical ectopic pregnancy with transvaginal ultrasound guided aspiration and single dose methotrexate. Fertil Steril 2004;81(4): 1130-1132.

9. Benson C B, Doubilet P M,Strategies for conservative treatment of cervical ectopic pregnancy. Ultrasound Obstet Gynaecol 1996;8:371-372.

10. Ushakov F B, Elchalal U, Aceman P J,Schenker J G, Cervical pregnancy : past and future. Obstet Gynaecol Surv 1997;52:45-59.

11. Tenor JL, Ectopic pregnancy, Am Fem Physician.2000;61(4):1080-1088.

12. Ginsberg ES, Frates MC,Rein MS. Early diagnosis and treatment of cervical pregnancy in an in vitro fertilization program. Fertil Steril.1994;61: 966-969.

13. Weyerman PC, Verhoeven ATM, Alberda AT: cervical pregnancy after in vitro fertilization and embryo transfer. Obstet Gynaecol, 1989;161:1145-1146.

14. Marcovici I, Rosenweig BA, Brill AI, Khan M, Scommagna A, cervical pregnancy. Obstet Gynaecol Surv, 1994;49;49-55.

15. Raskin MM,. Diagnosis of cervical Pregnancy by ultrasound. A case report. Am J Obstet Gynecol 1978;130; 234-235.

16. Timor-Tritsch IE, Monteagurdo A, Mandeville EO, et al Successful management of viable cervical pregnancy by local injection of methotrexate guided by transvaginal ultrasonography. Am J Obstet Gynecol. 1994;17:737-739.

17. Frates MC, Benson CB, Doubilet PM, et al. Cervical ectopic pregnancy. Radiology. 1984; 191:773-775.

18. Vas W, Suresh PL, Tang-Brton et al. Ultrasonographic differentiation of cervical abortion from cervical pregnancy, J Clin ultrasound. 1984; 12:553-537.

19. Jurkovic D, Hacket E Campbell S. Diagnosis and treatment of early Cervical pregnancy. Ultrasound Obstet Gynecol. 1996;8:373-380.

20. Qasim SM, Bohrer MK, Kemmann E. Recurrent Cervical pregnancy after assisted reproduction by intra-fallopian transfer. Obstet Gynaecol 1996;87:831-832.

21. Condous G, Okaro e, Khalid A, Lu C, Van Huffel S, Timmerman D, Boure t. A prospective evaluation of a single-visit strategy to manage pregneies of unknown location. Hum Reprod 2005;20;1398-1403.

22. Kirk E, CondousG, Haider Z, Syed A, Ojha K. and Bourne T. The Conservative management of cervical ectopic pregnancies. Ultrasound Obstet Gynecol. 2006; 27(4): 430-43 7.

23. Stovall TG, Ling FW. Single dose methotrexate: an expanded clinical trial. Am J obstet Gynecol 1993; 168: 1759-1765.

24. Hochner-Celnikier D, Ron M, Goshen R, Zacut D, AmirG, Yagel S. Rupture of ectopic Pregnancy following disappearance of serum beta subunit of hCG. Obstet Gynecol 1992; 79:826-827.

25. Hung TH, Shau WY, Hsieh TT, Hsu JJ, Soong YK, Jeng CJ Prognostic factors for an unsatisfactory Primary Methorexate treatment of Cervical Pregnancy: a quantitive review. Hum Repod 1998; 2636-2642.

26. Msogitis S, Pilalis a, Daskalakis G, Papantoniou N, Antsaklis A. Management of early viable cervical ectopic pregnancy: results of Conservative treatment. Radiology 1994:191:773-775.

27. Ushakov FB, Elchalal U, Aceman PJ, Schenker JG. Cervical Pregnancy: past and future. Obstet Gynecol Surv. 1996;52:45-59. 